\title{
Tecnologias no trabalho docente: um olhar para o processo cognitivo de construção de estratégias pedagógicas
}

Ana Beatriz Michels, UFRGS, ana.michels@ufrgs.br, 0000-0001-6813-2622

Ângela de Moura Ferreira Danilevicz, UFRGS, angelamfd@ producao.ufrgs.br, 0000-00034518-9567

Rosane Aragón, UFRGS, rosane.aragon@ufrgs.br, 0000-0002-0307-4457

\section{Resumo}

O contexto educacional vigente vem exigindo das instituições de ensino e dos docentes uma busca constante pela inovação, sendo necessário um repensar da formação continuada e, em especial, da prática pedagógica. O presente artigo propõe um modelo para compreensão do processo cognitivo de construção de estratégias educacionais pelos docentes. O modelo foi construído com base no constructo Piagetiano dos possíveis, a partir de uma pesquisa exploratória, de natureza qualitativa, na qual foram analisadas as vivências de docentes em duas arquiteturas pedagógicas aplicadas numa formação continuada online. Através de um processo evolutivo e não-linear, o modelo permite ao docente buscar heurísticas para abrirse a novos possíveis, de acordo com os desafios que vivencia, estimulando-o a se reinventar em prol de uma educação emergente.

Palavras-chave: estratégia pedagógica, arquitetura pedagógica, formação continuada online, possível cognitivo

\section{Technologies in teaching work: a look at the cognitive process of construction pedagogical strategies}

\begin{abstract}
Constant research for different teaching tools and methods, considering the demand for continued education and innovative pedagogical practices, is the challenge encountered by educational institutions and educators in facing the present context. This paper aims to propose a model for understanding the cognitive process of constructing educational strategies applied in online undergraduate courses. The model was built on the Piagetian of possible, from an exploratory research, of a qualitative nature, about the professors experiences in two pedagogical architectures applied in a continuous online training. Through an evolutionary and non linear process, the modelo allows professors to search for heuristics to open up to new possibilities, according to the challenges they face, encouraging them to recreate pedagogical practices for a differentiated education.
\end{abstract}

Keywords: pedagogical strategy, pedagogical architecture, online continuing education, cognitive possible

\section{Introdução}

A sociedade está em constante reconstrução, o que impacta significativamente nas organizações, especialmente em relação à dinâmica, flexibilidade e inovação para atender às suas demandas. Nesse cenário, a Universidade - vista "como um lugar para ampliar a mente, para fazer contatos e aprender a aprender" - é um dos órgãos impactados, devendo repensar suas ideias acadêmicas (UFRGS, p. 26, 2016). Esse repensar vem exigindo das instituições de ensino, em destaque dos docentes, um aprimoramento de seus modelos e práticas 
pedagógicas. Os docentes são confrontados com a "inevitabilidade de serem criativos e inovadores [...]" (Gonçalves, Fonseca, Malça, 2016, p. 09).

Neste processo de inovação (Walder, 2017), os docentes precisam desenvolver estratégias pedagógicas significativas e coerentes com os desafios do mundo. Eles passam de transmissores para mediadores do conhecimento e os alunos assumem uma postura de protagonistas perante o processo de aprendizagem. Tendo a aprendizagem ativa (Debald, 2020; Valente, Almeida, Geraldini, 2017; Ghilay, Ghilay, 2015) como um novo paradigma, há uma maior valorização pelo aprender do que pelo saber, oportunizando um aprendizado constante dos alunos e o desenvolvimento de soluções inovadoras para os desafios enfrentados pela sociedade.

O desafio atual, ocasionado pelo isolamento social, fruto da pandemia do Covid-19 e, consequentemente, pelos modelos de ensino adotados por diferentes instituições de ensino superior, desequilibrou - e segue desequilibrando - os docentes, que precisam se adaptar, às pressas, à nova realidade. Eles foram “apanhados de surpresa” e, mesmo que o potencial de resposta à crise atual está muito mais nos docentes do que nas instituições ou políticas (Nóvoa, Alvim, 2021), há urgência em desenvolver processos educativos visando a melhoria da qualidade profissional dos mesmos, em termos de formação e capacitação docente em prol de uma educação digital em rede (Moreira; Schlemmer, 2020, p. 28).

Analisando este cenário enquanto desafios e oportunidades em prol de uma educação inovadora, percebe-se que a formação docente precisa ser pensada para além da instrumentalização (Oliveira, Corrêa, Morés, 2020), caracterizada não só pela reflexão docente acerca da sua prática em sala de aula, mas pelo diálogo e construção entre pares. Por esse viés, o docente ressignifica o seu fazer pedagógico.

A ressignificação envolve um processo de reflexão sobre a ação e uma ação atualizada que mostra - ou não - a abertura para um conjunto de novos possíveis (Piaget, 1985). E o conjunto de potenciais novos possíveis é apresentado por Nóvoa e Alvim (2020) quando afirmaram a importância do compartilhamento de ideias e projetos inovadores que são construídos na colaboração entre docentes da mesma instituição de ensino ou com outras instituições. Um dos destaques é a ação da UNESCO (2020) que, frente à pandemia ocasionada pelo Covid-19, apresentou plataformas e ferramentas digitais utilizadas globalmente. Porém, para que essas ideias, enquanto estratégias pedagógicas, sejam replicadas, ampliadas e reinventadas, cada docente precisa tomar consciência do seu processo cognitivo na busca pela inovação pedagógica para que, posteriormente, aplique-as em suas aulas, sejam elas presenciais ou virtuais.

Com base nesse contexto, este artigo tem como questão norteadora o entendimento de como ocorre o processo cognitivo de docentes na construção de estratégias pedagógicas num contexto de formação continuada. $\mathrm{O}$ objetivo da pesquisa ${ }^{1}$ está em propor um modelo para compreensão do processo cognitivo de construção de estratégias pedagógicas, de docentes do ensino superior.

\section{Formação e Profissão docente numa perspectiva interacionista}

As transformações na educação fazem emergir questionamentos de como é possível inovar, buscando abordagens que possam modelar o hoje e o futuro. Enxergando que a inovação impacta positivamente na melhora da educação, o seu conceito exige algumas características por parte dos atores envolvidos, em especial dos docentes. Dentre as características, estão $(i)$ uma certa insatisfação e inconformidade com o que existe, buscando respostas para se introduzir alguma novidade; (ii) criatividade para se incorporar algo novo; 
(iii) um olhar positivo da instituição, com perspectivas de melhora; (iv) ter paixão, compromisso e entusiasmo (Guerra, 2020).

Mesmo sabendo que há distinção entre inovação pedagógica e inovação tecnológica (Walder, 2017), o docente deve ser - ou se tornar - "[...] um profissional crítico, reflexivo e competente para o domínio das novas tecnologias digitais" (Garcia et al, 2011, p. 80).

Há uma década esses autores afirmavam que a formação do profissional docente implica no "[...] uso das tecnologias não de forma exógena à docência, mas inerente a ela e necessário ao processo abrangente de formação integral do ser humano" (Garcia et al, 2011, p. 80). E, atualmente, ainda persiste a necessidade de mudança organizacional por um olhar disruptivo. Uma pesquisa recente mostrou que, dentre os desafios postos pela pandemia, "[...] a escassez da formação continuada docente é uma realidade muito presente nas Instituições de Ensino Superior (IES), com números que somam 44,3\% raras formações ou nenhuma formação" (Silus, Fonseca, Jesus, 2020, p. 14). Mesmo que a necessidade tenha prevalecido sobre a inércia, as soluções encontradas para a educação, conforme afirmam Nóvoa e Alvim (2020), ainda são frágeis e precárias.

As mudanças necessárias, frente à atual sociedade globalizada, envolvem desafios pessoais e coletivos de transformação e inovação, com foco na formação docente ao longo da vida, "[...] que realcem a realidade multifacetada, multidimensional, multidisciplinar e multicultural, assim como a articulação de saberes que se exige aos atuais professores/formadores [...]" (Moreira, Schlemmer, 2020, p. 17).

Pelo viés do modelo educacional relacional/interacionista, vigente na era atual da sociedade digital e em rede, o uso de arquiteturas pedagógicas é uma das possibilidades de estimular a inovação por parte dos docentes, impactando na sua aprendizagem e de seus alunos. Enquanto estratégias de aprendizagem, as arquiteturas pedagógicas conectam uma abordagem pedagógica com uma teoria explicitada e práticas criativas, propondo "[...] deslocamentos que visam dar às tecnologias sentidos que ultrapassem o seu uso periférico e dissociado das práticas pedagógicas, a fim de explorar novas possibilidades de referenciamento dos espaços e tempos" (Aragón, 2016, p. 263).

No processo de construção de arquiteturas pedagógicas por parte dos docentes, é possível se embasar em propostas já conhecidas e compartilhadas em comunidades de aprendizagem, plataformas, redes sociais, revistas, livros, cursos, etc. E essas propostas de estratégias pedagógicas articulam e organizam "[...] as condições de ensino para que possa se construir aprendizagens concretas, ou seja, considerando além dos processos de ensinar, também os processos de aprender" (Martins, Giraffa, Lima, 2018, p. 02). E quanto maior for o repertório de estratégias do docente, maior a probabilidade dele poder ser criativo e inovar na sua prática pedagógica em sala de aula.

E nesse processo de inovar, a prática pedagógica é vista como o "saber docente aplicado em sala de aula com o uso de recursos, sejam eles tecnológicos, didáticos, materiais ou vivenciais, dentre outros, com foco no aprendizado do aluno, conferindo significado aos processos de ensino e aprendizagem" (Michels, Danilevicz, 2020). Nesse sentido, a prática pedagógica docente é inovadora no momento em que o uso de recursos, tecnológicos ou analógicos, são sustentados por metodologias ativas e por um aporte teórico.

Analisando o processo constante e crescente de produção de novidades, Piaget (1985) afirmou que esse processo está centrado na questão da formação dos "possíveis", que é o produto da construção do sujeito na interação com as propriedades do objeto. E nesse processo sempre é possível incorporar coisas novas, levando à formação "[...] não somente de novos possíveis imediatamente atualizáveis, mas daquilo que se poderia chamar um 
"campo virtual de possibilidades"” (Piaget, 1985, p. 136). Ou seja, no momento que o sujeito resolveu certo desafio, buscando uma heurística para solucioná-lo, caso ele se depare com situações inteiramente novas, será possível buscar outras heurísticas. E nessas novas situações, pelo fato do sujeito ainda não imaginar os possíveis que poderá constituir, o "campo virtual" são as possibilidades "[...] de descoberta ou de diferenciação de novos possíveis" (Piaget, 1985, p. 136).

A abertura para "novos possíveis" depende das interpretações de cada sujeito/docente em relação às suas atividades. E, enquanto formação docente numa perspectiva interacionista, o compartilhamento de estratégias pedagógicas oportuniza a construção de um leque de possibilidades a serem aplicadas em sala de aula.

\section{Percurso Metodológico}

Esta pesquisa exploratória propôs um estudo de caso de natureza qualitativa (Yin, 2016), em que o interesse está nas perspectivas dos participantes, com um olhar para suas concepções, práticas e conhecimento relativo à questão em estudo (Flick, 2011). O percurso metodológico envolveu quatro etapas, que são detalhadas após a contextualização do caso.

O caso envolveu a formação continuada online intitulada SOS Professor: Práticas Pedagógicas Inovadoras no contexto de pandemia, ofertada para docentes da Universidade Federal do Rio Grande do Sul. A formação foi planejada e realizada no início da pandemia ocasionada pelo Covid-19. O SOS Professor fez parte de uma trilha de aprendizagem de cursos realizados pela Escola de Desenvolvimento da UFRGS - EDUFRGS voltados ao aperfeiçoamento dos docentes no que tange o olhar pedagógico e tecnológico do uso emergencial de ferramentas digitais para o contexto do Ensino Remoto Emergencial - ERE.

Enxergando para além da pandemia e além de uma formação instrumental das ferramentas digitais, o propósito do SOS Professor foi oportunizar a melhoria do fazer pedagógico do docente em sala de aula a partir da reflexão, compartilhamento e reconstrução de estratégias pedagógicas que possam ser utilizadas nos contextos presencial e virtual.

A sequência didática do curso envolveu a construção e aplicação de arquiteturas pedagógicas (Carvalho, Nevado, Menezes, 2007), enquanto estratégias de aprendizagem que integram um olhar amplo, conectando as tecnologias - sejam elas digitais ou analógicas com modelos pedagógicos. Elas foram desenvolvidas e aplicadas ao longo da formação, permitindo uma experimentação por parte dos alunos-docentes e uma ampliação do leque de estratégias pedagógicas a serem utilizadas pelos mesmos em suas aulas. Com essa proposta, os alunos-docentes participaram do curso online numa perspectiva de aluno e de docente.

A primeira etapa do percurso metodológico se constituiu na identificação das arquiteturas pedagógicas para oportunizar uma reflexão dos docentes acerca de estratégias educacionais. A primeira, intitulada Vitrine de Práticas Pedagógicas no contexto de pandemia, perpassou todos os encontros do SOS Professor. Ela foi desenvolvida com o objetivo de estimular a construção, em várias mãos, de um portfólio de estratégias de ensino e aprendizagem que possam ser copiadas ou reconstruídas para a realidade de cada professor. Para a construção desse portfólio foi utilizado a ferramenta digital Padlet, que é um mural digital que estimula a colaboração e a produtividade.

A proposta dessa arquitetura era que, ao término de cada encontro síncrono online, cada aluno-docente compartilhasse no Padlet uma reconstrução de alguma atividade que foi realizada no encontro. A ideia da reconstrução envolvia tanto um olhar de cada aluno-docente para contextos diferentes - em especial nas suas áreas de conhecimento, quanto ao uso de 
recursos tecnológicos diferentes e outras situações em sala de aula (momentos síncronos ou assíncronos).

A segunda arquitetura pedagógica, intitulada Como posso recriar uma estratégia pedagógica no meu contexto?, foi utilizada nos três últimos encontros do SOS Professor, focado no compartilhamento de estratégias educacionais. A proposta era que, em cada um dos encontros síncronos online, dois alunos-docentes compartilhassem alguma estratégia que tivessem aplicado em sala de aula. E o desafio era que a aplicação da estratégia fosse pelo viés interacionista, ou seja, que não fosse num formato de apresentação, e sim de experimentação pelos demais alunos-docentes.

Após a vivência dos alunos-docentes em cada estratégia pedagógica compartilhada, cada um deles tinha que pensar em como recriá-la para o seu contexto, nas suas disciplinas, nas suas aulas. Para auxiliar, foi utilizado o Google Docs para criar um documento com um espaço definido para cada aluno-docente descrever de que forma recriaria cada uma das seis estratégias pedagógicas compartilhadas.

A segunda etapa do percurso envolveu a caracterização dos sujeitos da pesquisa, que foram os 35 docentes $^{2}$ da UFRGS, de diferentes áreas, selecionados para participar do SOS Professor. A escolha dos participantes com formações distintas corrobora com a visão de Nóvoa (2020) que afirmou que ser professor é uma profissão coletiva e que as melhores respostas à pandemia vieram daqueles professores que em conjunto, uns com os outros, colaboraram com as respostas.

Após a caracterização dos sujeitos da pesquisa, a terceira etapa do percurso metodológico se constituiu no levantamento e análise das condutas cognitivas dos docentes ao longo de suas participações nas duas arquiteturas pedagógicas apresentadas anteriormente. A análise foi desenvolvida com base na teoria Piagetiana dos possíveis e os dados foram organizados com o apoio do software Nvivo $^{3}$. A partir dos resultados da terceira etapa, a quarta etapa consistiu na proposição do modelo para compreensão do processo cognitivo de construção de estratégias pedagógicas, de docentes do ensino superior.

\section{Análise dos Dados e Discussão dos Resultados}

As condutas cognitivas dos sujeitos, analisadas nas suas produções nas duas arquiteturas pedagógicas contextualizadas, envolveram uma compreensão dos sujeitos sobre as estratégias educacionais que foram compartilhadas ao longo do SOS Professor. Os alunosdocentes foram desafiados a enxergarem - e compartilharem nas arquiteturas pedagógicas possibilidades de implementação e aprimoramento das mesmas em seus contextos pedagógicos.

Pelo fato do constructo Piagetiano dos possíveis afirmar que um possível cognitivo é a própria invenção e criação dos sujeitos (Piaget, 1985), as condutas cognitivas dos sujeitos foram analisadas à luz dessa teoria. Os dados - intervenções dos sujeitos nas arquiteturas pedagógicas - mostraram regularidades cognitivas que são contextualizadas a seguir.

Num primeiro olhar, alguns extratos mostraram que os sujeitos não concebem possibilidades de uso e nem de reconstrução de novas estratégias pedagógicas compartilhadas por outros docentes - em seus contextos. Vista como uma pseudoimpossibilidade, este processo cognitivo está relacionado tanto aos saberes tecnológicos, quanto aos pedagógicos e à prática criativa por parte do sujeito.

Para exemplificar, o sujeito S5, da área da Psicologia, compartilhou uma estratégia pedagógica que envolveu o uso do recurso tecnológico Socrative para uma atividade de gamificação de construção prévia de conhecimento. Buscando uma recriação desta estratégia, 
o sujeito S7, da área do Jornalismo e Museologia, publicou, em uma das arquiteturas pedagógicas, que "Talvez por ter tido dificuldade com a ferramenta e também com a atividade, não vejo como apropriá-la nas minhas disciplinas". Percebe-se que o uso de recursos tecnológicos e pedagógicos são dificultadores na apropriação da estratégia por parte do S7.

Após um olhar de pseudo-impossibilidade, as demais produções dos sujeitos nas duas arquiteturas pedagógicas mostraram que eles concebem possibilidades de implementação e posteriormente aprimoramento - das estratégias pedagógicas compartilhadas no SOS Professor.

Para um olhar de implementação, alguns sujeitos simplesmente reproduzem novas estratégias pedagógicas como uma receita, apenas adaptando-as ao seu contexto. Eles repetem todas as etapas de alguma estratégia que foi compartilhada por outro docente. Nesta situação, a compreensão do sujeito encontra-se num momento de co-possível analógico, como é o caso do sujeito S6, da área de Genética, que apresentou como fazer uma enquete na plataforma Teams, a partir de um vídeo que gravou no QuickTime e converteu para MP4. No vídeo ele fez um tutorial das etapas, com imagens, e, pela transcrição, ele comentou que “[...] Dentro do Teams, a gente vai em Postagens, clica nos três pontinhos e clica em Forms. $E$ aí podemos fazer a nossa pergunta, inserir as opções, permitir mais de uma resposta por pessoa, ou não. Aí dou o avançar e enviar. Aí ele aparece para todos votarem dentro das postagens". O sujeito apenas apresentou o olhar tecnológico do recurso, sem apresentar outros saberes docentes necessários para uso em sala de aula.

Para um olhar inicial de aprimoramento das estratégias pedagógicas compartilhadas no SOS Professor, alguns sujeitos fizeram adequações em relação a estas estratégias, trazendo novos recursos tecnológicos ou pedagógicos. Essas adequações, enquanto abertura para um novo possível, são vistas como um co-possível concreto, havendo uma progressão inicial de um procedimento analógico para um dedutível (Piaget, 1985). Os extratos mostraram indícios iniciais de um olhar de prática criativa por parte do sujeito, mas ainda coordenados com a estrutura da estratégia inicial.

O sujeito S19, da área da Comunicação, apresentou uma estratégia com um novo olhar pedagógico para o uso do podcast em sala de aula. "Usaria na minha disciplina de Projetos culturais. Num primeiro momento apresentando rápidos conceitos, depois, como apresentamos no grupo, também solicitaria podcast sobre textos que os alunos apresentariam como seminário."

Para um olhar mais amplo de aprimoramento de estratégias pedagógicas, alguns extratos mostraram que os sujeitos concebem outros recursos, possibilidades e/ou abordagens para alcançar o mesmo objetivo da estratégia pedagógica compartilhada por outros docentes no curso, considerando aspectos pedagógicos, tecnológicos e a prática criativa. Nesta situação, as atualizações são vistas como co-possíveis abstratos, envolvendo uma inovação incremental. E essas atualizações passam a ser mais amplas e numerosas em relação ao momento descrito anteriormente.

Para exemplificar, o sujeito S5 apresentou uma dinâmica para formação de grupos online, usando dois recursos tecnológicos. "Como realizar a dinâmica de formação de grupos: 1) Utilizando o aplicativo "Random name picker", o professor sorteia os alunos que serão os representantes de cada grupo (no caso de 6 grupos, sortear 6 alunos, e assim por diante). 2) Cada aluno, identificando-se apenas com o seu número de matrícula, irá se descrever, no Padlet, como se estivesse se candidatando para um emprego, mas o conteúdo 
deve ser verdadeiro. 3) Cada representante (alunos sorteados) seleciona os integrantes do seu grupo a partir da escolha de cada "post-it" do mural criado no Padlet."

Entendendo que o aprimoramento de estratégias pedagógicas pode oportunizar a busca pela inovação disruptiva, o constructo Piagetiano dos possíveis mostra que, enquanto co-possíveis ilimitados, os sujeitos podem enxergar possibilidades de construção de novos recursos tecnológicos e pedagógicos, a partir do compartilhamento de estratégias pedagógicas por outros docentes. Nesta situação, há generalização relacionada a qualquer procedimento envolvendo uma determinada estratégia pedagógica, mesmo que o sujeito ainda não saiba os meios para alcançar o resultado (Piaget, 1985). Porém, nenhuma das produções dos sujeitos nas arquiteturas pedagógicas mostraram indícios de generalização.

A partir da análise sustentada na teoria Piagetiana dos possíveis, o modelo do processo cognitivo de construção de estratégias pedagógicas é representado na Figura 1. O modelo é a maneira como o sujeito internaliza e compreende o uso de estratégias pedagógicas, num processo cognitivo de construção dos possíveis. Esse processo cognitivo ocorre em níveis, envolvendo reflexão-ação do sujeito acerca de desafios vivenciados.

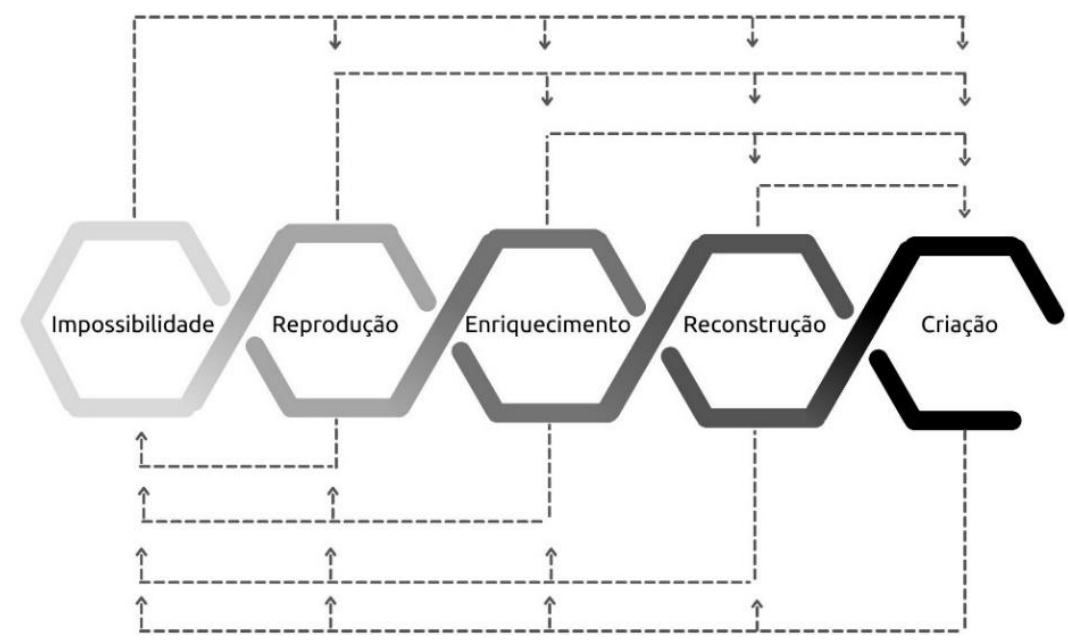

Figura 1 - Processo cognitivo de construção de estratégias pedagógicas

Nível I - Impossibilidade: relaciona-se com o nível de pseudo-impossibilidade em que, diante de determinado desafio, o sujeito, não tendo conhecimento e/ou prática acerca do mesmo, se sente incapaz de usar a estratégia pedagógica em suas aulas.

Nível II - Reprodução: relaciona-se com o nível do possível analógico em que, diante de alguma perturbação, o sujeito se sente capaz de apenas reproduzir alguma estratégia pedagógica que já foi compartilhada por terceiros - de forma presencial ou virtual - apenas trazendo para o seu contexto.

Nível III - Enriquecimento: relaciona-se com o nível do co-possível concreto em que, diante de determinado desafio, o sujeito se sente capaz de fazer adequações em relação a alguma estratégia pedagógica que já foi compartilhada por terceiros, adaptando-a para o seu contexto.

Nível IV - Reconstrução: relaciona-se com o nível de co-possíveis limitados em que, diante de alguma perturbação, o sujeito, tendo conhecimento e/ou prática de outros recursos, possibilidades e/ou abordagens, usa-os para reconstruir uma estratégia pedagógica, de formas diversas, mas ainda limitadas. 
Nível V-Criação: relaciona-se com o nível de co-possíveis ilimitados em que, diante de determinado desafio, o sujeito usa seu conhecimento prévio acerca de outros recursos, possibilidades e/ou abordagens para criar novos recursos tecnológicos e pedagógicos e, consequentemente, novas estratégias pedagógicas.

O modelo mostra um processo cognitivo, evolutivo e não-linear que, de acordo com a abordagem relacional/interacionista, o sujeito sempre constrói conhecimento a partir de suas experiências anteriores e de novos desafios que surgem. Dependendo do desafio vivenciado, o sujeito poderá: ( $i$ ) retomar o nível da impossibilidade, até buscar uma heurística, alcançando o nível de abertura para esse "novo possível” ou ( $i i)$ retomar qualquer um dos níveis anteriores, concebendo possibilidades de reprodução, enriquecimento, reconstrução ou criação, não necessariamente nesta ordem e de acordo com as possibilidades do seu "campo virtual".

Hoje, depois de mais de um ano no modelo de ERE, utilizado pela UFRGS, muitos docentes enxergam-se num nível de enriquecimento e/ou reconstrução de estratégias pedagógicas para suas aulas, podendo até alcançar o nível de criação. Porém, muitos destes docentes, há alguns meses atrás, se sentiam nos níveis de impossibilidade e/ou reprodução de estratégias pedagógicas. Por outro lado, esses docentes que hoje estão no nível de reconstrução de estratégias pedagógicas para uso no ERE, frente a desafios ainda mais complexos, como exemplo a realidade aumentada, ainda não sabem como aplicá-la no ensino presencial, a distância ou híbrido.

Através do modelo do processo cognitivo de construção de estratégias pedagógicas, busca-se ilustrar como ocorre o processo de ressignificação de estratégias educacionais planejadas e aplicadas pelos docentes em suas aulas. $\mathrm{O}$ modelo pode ser utilizado pelos docentes em todos os momentos do seu fazer pedagógico - planejamento, execução e avaliação - permitindo uma metareflexão constante e uma busca por formações continuadas que foquem na aprendizagem ativa dos participantes.

\section{Considerações Finais}

O contexto educacional vigente, resultante da pandemia do Covid-19, vem exigindo do docente um papel de provocador e mediador, ao mesmo tempo que inovador na sua prática pedagógica. E esse olhar da prática pedagógica docente envolve a busca por estratégias que permitam um aprendizado constante dos alunos e o desenvolvimento de soluções inovadoras para os desafios enfrentados pela sociedade. Nesse sentido, a pesquisa teve como objetivo propor um modelo para compreensão do processo cognitivo de construção de estratégias pedagógicas, de docentes do ensino superior.

O modelo foi desenvolvido a partir da análise das vivências de docentes em duas arquiteturas pedagógicas aplicadas na formação continuada online SOS Professor: Práticas Pedagógicas Inovadoras no contexto de pandemia, com base na teoria Piagetiana dos possíveis. O modelo estimula um olhar atual do fazer pedagógico do docente que, de acordo com seus desafios vivenciados, pode buscar heurísticas que o permita abrir-se para novos possíveis em relação a estratégias pedagógicas. A universidade tem um papel essencial nesse processo de abertura de novas possibilidades para a docência, através do desenvolvimento constante de formações continuadas que incentivem o compartilhamento de experiências e a construção entre pares.

Para um olhar de futuras pesquisas, que serão abordadas nas próximas etapas da tese de uma das autoras, pretende-se analisar o "campo virtual" de possibilidades dos docentes como uma "trilha virtual de aprendizagem", para além da formação continuada. Como os 
docentes têm construído suas "trilhas virtuais de aprendizagem" e aplicado na sua prática em sala de aula?

O processo cognitivo de abertura de novos possíveis conduz à inovação em sala de aula, mediante a criação e aplicação de estratégias pedagógicas. E estas estratégias, quando imersas e conectadas com a sociedade atual, digital e em rede, envolvem o uso de tecnologias para além de um olhar instrumental, essencial para que os docentes tenham uma prática criativa. E nesse processo, os indícios de inovação incremental e disruptiva são vislumbrados e os docentes passam a se reinventar perante os desafios de uma educação emergente.

\footnotetext{
Notas

${ }^{1}$ A pesquisa está relacionada com a primeira etapa da proposta de tese de uma das autoras, que tem como tema central a busca pela inovação pedagógica no ensino superior, com olhares para a concepção e prática docente. ${ }^{2} \mathrm{Na}$ análise dos dados, os 35 sujeitos foram apresentados como $\mathrm{S} 1$ até S35, sendo que a ordem foi definida de forma aleatória.

${ }^{3} \mathrm{O}$ software NVivo auxilia na organização e análise dos dados da pesquisa. Está disponível em https://www.qsrinternational.com/nvivo-qualitative-data-analysis-software/home.
}

\section{Referências Bibliográficas}

ARAGÓN, R. Interação e mediação no contexto das arquiteturas pedagógicas para a aprendizagem em rede. In: Revista de Educação Pública, v. 25, n. 59/1, p. 261-275. UFMT, 2016. Disponível em <https://www.lume.ufrgs.br/handle/10183/150441>. Acesso em: 23 jul. 2021.

CAMARGO, F.; DAROS, T. A sala de aula inovadora: estratégias pedagógicas para fomentar o aprendizado ativo. Porto Alegre: Penso, 2018.

DEBALD, B. Metodologias ativas no ensino superior: o protagonismo do aluno. Penso Editora, 2020.

FLICK, U. Desenho da pesquisa qualitativa. Porto Alegre: Artmed, 2011.

GARCIA, M. F. et al. Novas competências docentes frente às tecnologias digitais interativas. Teoria e Prática da Educação, v. 14, n. 1, p. 79-87, 2011. Disponível em: $\langle$ https://periodicos.uem.br/ojs/index.php/TeorPratEduc/article/view/16108>. Acesso em: 08 abr. 2021.

GHILAY, Y.; GHILAY, R. TBAL: Technology-based active learning in higher education. Ghilay, Y. \& Ghilay, p. 10-18, 2015. Disponível em: <https://papers.ssrn.com/sol3/papers.cfm?abstract_id=3736634>. Acesso em: 12 abr. 2021.

GONÇALVES, S.; FONSECA, P.; MALÇA, C.. Inovação no ensino superior. 2016.

GUERRA, M. Á. S. Innovar o morir. La Orientación en la mejora del desarrollo y bienestar personal. Universidad de La Rioja, p. 15-33, 2020.

MARTINS, C.; GIRAFFA, L. M. M.; LIMA, V. M. Gamificação e seus potenciais como estratégia pedagógica no ensino superior. Renote, v. 16, n. 1, 2018. Disponível em: 〈https://www.seer.ufrgs.br/renote/article/view/86005 >. Acesso em: 08 abr. 2021. 
MICHELS, A. B.; DANILEVICZ, A. F. M. SOS Professor: Práticas Pedagógicas Inovadoras no contexto de pandemia. Escola de Desenvolvimento de Servidores da UFRGS, 2020.

MOREIRA, J. A.; SCHLEMMER, E. Por um novo conceito e paradigma de educação digital onlife. Revista uFG, v. 20, 2020. Disponível em: <https://www.revistas.ufg.br/revistaufg/article/view/63438 >. Acesso em: 08 abr. 2021.

NÓVOA, A. Formação de professores em tempo de pandemia. Instituto Yungo, 2020. Disponível em: <https://www.youtube.com/watch?v=ef3YQcbERiM\&t=2s >. Acesso em: 20 mar. 2021.

NÓVOA, A.; ALVIM, Y. Nothing is new, but everything has changed: A viewpoint on the future school. Prospects, v. 49, n. 1, p. 35-41, 2020. Disponível em: <https://link.springer.com/article/10.1007/s11125-020-09487-w>. Acesso em: 29 abr. 2021.

OLIVEIRA, R. M.; CORRÊA, Y.; MORÉS, A. Ensino remoto emergencial em tempos de covid-19: formação docente e tecnologias digitais. Revista internacional de formação de professores, volume 5, p. 1-18, 2020. Disponível em: <https://periodicoscientificos.itp.ifsp.edu.br/index.php/rifp/article/view/179>. Acesso em: 08 mar. 2021.

PIAGET, J. O possível e o necessário: evolução dos possíveis na criança. Porto Alegre, Artes Médicas, 1985.

SILUS, A.; FONSECA, A. L. C.; JESUS, D. L. N. Desafios do ensino superior brasileiro em tempos de pandemia da COVID-19: repensando a prática docente. Liinc em Revista, v. 16, n. 2, p. e5336-e5336, 2020. Disponível em: 〈http://revista.ibict.br/liinc/article/view/5336>. Acesso em: 08 mar. 2021.

UNESCO. UNESCO's support: Educational response to COVID-19. Disponível em: <https ://en.unesc o.org/covid 19/ educa tionr espon se/suppo rt>. Acesso em: 14 mai. 2021. Universidade Federal do Rio Grande do Sul. Plano de Desenvolvimento Institucional: PDI 2016 - 2020: Construa o futuro da UFRGS. Porto Alegre: UFRGS, 2016. Disponível em: <http://www.ufrgs.br/pdi/PDI_2016a2026_UFRGS.pdf $>$. Acesso em: 08 de mar. 2021.

VALENTE, J. A.; ALMEIDA, M. E. B.; GERALDINI, A. F. S.. Metodologias ativas: das concepções às práticas em distintos níveis de ensino. Revista Diálogo Educacional, v. 17 , n. 52, p. 455-478, 2017. Disponível em: <https://www.redalyc.org/pdf/1891/189154955008.pdf > . Acesso em: 08 mar. 2021.

WALDER, A. M. Pedagogical Innovation in Canadian higher education: Professors' perspectives on its effects on teaching and learning. Studies in Educational Evaluation, v. 54, p. 71-82, 2017. $\quad$ Disponível em https://www.sciencedirect.com/science/article/abs/pii/S0191491X16300153>. Acesso em: 08 mar. 2021.

YIN, R. K. Pesquisa qualitativa do início ao fim. Porto Alegre, RS: Penso, 2016. 